TRANSFER RNA

\section{Search for Recognilion}

from our Molecular Biology Correspondent

THE sport of identifying the various functional sites on tRNA, by selective chemical modification, by studying activity in heterologous systems or, less fatiguingly, by looking for correlations between specificity and features of the numerous sequences now available for contemplation, has in general proved rather disappointing. In particular there is hardly any part of the structure that has not been implicated in the specific interaction with the aminoacyl tRNA synthetase.

A possible, though unpleasing, explanation is that the recognition mechanisms of different tRNAs for their particular synthetases have little in common. Workers in the field are not, however, as it seems, prepared to give up without a struggle, and attention is now being focused on one nucleotide in tRNA, the fourth from the $3^{\prime}$ end. Roe and Dudock (Biochem. Biophys. Res. Commun., 49, 339 ; 1972) have homed in on this locus on the grounds of correlations that have emerged from heterologous charging experiments, in particular those in which a synthetase from one species aminoacylates the wrong tRNA from another. For instance, it has been known for some time that yeast phenylalanyl tRNA synthetase can operate on the phenylalanine tRNAs of various other species, as well as on a valine tRNA of Escherichia coli.

Roe and Dudock have now made a collection of nine $E$. coli tRNAs, all of which are charged by this same yeast synthetase. Aside from the nucleosides that are conserved in all tRNAs so far studied, the sequenced species that are aminoacylated by the synthetase, eight in number, all have twelve other nucleotides in common; eight of these nucleotides form the base-paired stem of the dihydrouridine loop of the clover leaf, one is in the loop itself, and another, an adenine, is the fourth residue from the $3^{\prime}$ end. The other, as yet unsequenced, chargeable tRNAs will provide a test of the inferences, and in one of them-one of the $E$. coli alanine tRNAs-Roe and Dudock have already looked for and found adenine in the fourth position.

In other tRNA species the sequences are very similar, indeed in three ( $E$. coli histidine, one of the $E$. coli glycine and yeast lysine tRNAs) all the critical residues for interaction with the yeast phenylalanine synthetase are there, except for the adenine at position 4 , and these are not aminoacylated. By the same token, species which do have adenine in this position, but lack the requirements in the dihydrouridine stem, are equally ineffective as substrates. The requirements may, of course, be narrowed down as new sequences come to light, but it seems entirely reasonable to infer that both regions of the molecule interact, at least with the synthetase in question.

Crothers, Seno and Söll (Proc. US Nat. Acad. Sci., 69, 3063; 1972) have simultaneously come up with a more explicit conjecture about the fourth nucleotide from the $3^{\prime}$ end. Accepting with evident reluctance that no single part of the sequence can be regarded as the recognition site, and that the dihydrouridine stem sequence does not, as several lines of evidence on mutant species indicate, tell the whole story, they have designed their own universal recognition system. They allocate to one site a preliminary sorting function of the tRNAs into four groups, one for each base. This they refer to as a "discriminator site". After being found acceptable by this site, the tRNA will interact with the synthetase by way of one or more further sites.

But how to choose a criterion for the subdivision into four groups? The somewhat primitive choice of chemical (not steric) nature of the amino-acid seems to meet the requirement, when matched against the base in the fourth position from the $3^{\prime}$ end. Actually Crothers et al. have been forced to add a fifth class, which slightly undermines the logic of their scheme, for whereas all other known tRNAs for any one amino-acid have a unique base in the critical position, the different arginine and lysine tRNAs from yeast have either a guanine or a uracil. For the rest, the correlation holds good, both for multiple tRNA of one amino-acid and between different species. Thus for the non-polar side chains (alanine, leucine, isoleucine, valine and phenylalanine) the fourth base is always adenine; it is also adenine in tyrosine, methionine and cysteine tRNAs, and arginine and lysine tRNAs of $E$. coli. For serine and threonine, as well as both glutamic and aspartic acids and their amides, the fourth base is guanine; that for histidine and proline is cytosine, and that for glycine is uracil. The random probability of such a correlation, though difficult to compute in any rationally quantitative manner, seems to be small, sufficiently so as to persuade the authors that their pattern is not a mirage. They suggest that the fourth base could be involved directly in recognizing the amino-acid or, more likely, may be an evolutionary survival from a primitive, myopic, recognition system which could discriminate only between chemically similar classes of amino-acids.

Concerning a different part of the tRNA chain, the pseudouridine loop, an interesting observation has been made by Simsek and RajBhandary (Biochem. Biophys. Res. Commun., 49, 508 ; 1972). They have completed the sequence of the initiator methionine tRNA from yeast, and find that the universal - $\mathrm{G}-$ $\mathbf{T}-\psi-\mathrm{C}-$ sequence is here replaced by $-\mathrm{G}-\mathrm{A}-\mathrm{U}-\mathrm{C}-$. The only other tRNA, in which the former sequence was reported not to occur, does not function in amino-acid incorporation. At another point in the same loop the initiator tRNA uniquely carries an adenine instead of a pyrimidine. The composition of the chain in this region presumably reflects the special function of the initiator.

\title{
New Assay for RD 114 Virus
}

THE intensive search that is currently being made for human RNA tumour viruses analogous to the RNA tumour viruses of lower mammals and birds has resulted in the isolation of RD 114 virus, a virus that has the biochemical and biophysical hallmarks of a typical C-type RNA virus but which has a unique intraspecies specific (group specific) antigenicity. This virus, which is released by human rhabdomyosarcoma cells after they had grown in the brain of a kitten, may yet prove to be the first human $\mathrm{C}$-type virus to have been isolated, and what Rand and Long have to say in Nature New Biology next week (December 6) should prove of interest to virologists working with RD 114 virus or related viruses.

Rand and Long set themselves the task of devising an assay for RD 114 virus and have come up with a syncytium formation assay which is essentially similar to the so-called XC cell assay for mouse leukaemia viruses. Rand and Long overlaid confluent monolayers of RD 114 cells liberating RD 114 virus with cells of a variety of human lines and then examined the cultures for syncytia. Cells of one line (KC cells), which are transformed by Rous sarcoma virus, formed abundant large syncytia either when they were co-cultivated with RD 114 cells or when they were exposed to purified stocks of RD 114 virus. By contrast, the untransformed parent cells from which KC cells were derived do not form syncytia in response to RD 114 virus or RD 114 cells.

Why and how $\mathrm{KC}$ cells respond to RD 114 virus by forming syncytia remains obscure; all that can be said at present is that the process depends upon intact RD 114 virus and treatment of the virus with either ether or detergents abolishes its ability to induce syncytia. But whatever the mechanism of the process proves to be the $\mathrm{KC}$ assay system provides not only an assay for RD 114 cells but also a method of screening for related viruses. 